\title{
p53 gene mutations, and CYP1A1 and GSTM1 genotypes in pulmonary squamous cell carcinomas
}

Susumu Ohshima, Ying Xu

\begin{abstract}
To investigate mechanisms causing p53 mutations in lung cancer cases, relations between $p 53$ gene mutations and aetiological factors such as smoking history or family history of cancers were studied in 35 primary lung cancer cases. The contribution of genotypes related to carcinogen metabolism (CYP1A1 and GSTM1) was also analysed. p53 mutations were observed in 13 cases $(37.5 \%)$. Seven $(53.8 \%)$ of the 13 patients with p53 mutation compared with five $(22.7 \%)$ of 22 patients without had a family history of cancer. However, there was no significant relation between p53 mutation or family history of cancer and CYP1A1 or GSTM1 genotypes. In conclusion, p53 mutation might be associated with the inherited characteristics that result in familial aggregation of lung cancer; however, this association was not explained by genotypes of enzymes related to carcinogen metabolisms. (F Clin Pathol: Mol Pathol 1997;50:108-110)
\end{abstract}

Keywords: lung cancer; p53 mutation; inherited factors

p53 gene mutations appear to be the most common genetic defects identified in human malignancies, including lung cancer, and they may be caused by a pathway through which environmental carcinogens initiate mutation. ${ }^{12}$ Although precise mechanisms causing p53 mutation are unknown, some investigators suggested a causal effect of carcinogens in tobacco smoke ${ }^{2}$ and inherited factors. ${ }^{1}$ There is no clear information on the possible aetiologic factors and genetic basis for p53 mutations in lung cancer. Among genetic factors that might be associated with lung cancer, genotypes CYP1A1 and GSTM1 have been linked to high risk of pulmonary squamous cell carcinoma, especially in the Japanese population. ${ }^{3}$ The polymorphic mutation of CYP1A1 gene from adenine to guanine in exon 7 is an amino acid substitution of isoleucine to valine (Ile $\rightarrow \mathrm{Val}$ ), and the prevalence of this genotype was significantly higher among lung cancer patients in Japan $^{3}$ and in a German group. ${ }^{4}$ Some studies have indicated a positive correlation between the GSTM1 null genotype and pulmonary carcinogenesis, especially squamous cell carcinoma. ${ }^{5}$ CYP1A1 and GSTM1 are involved in the biotransformation of tobacco derived polycyclic aromatic hydrocarbons (PAHs), and detoxification reactions, respectively. It is, therefore, postulated that individuals with a CYP1A1 mutation and GSTM1 null genotype should have a higher PAH-DNA adduct level in lung tissue, which can induce genetic mutations, such as in the p53 gene, that lead to an increased risk of cancer.

In this study, we investigated p53 gene mutations in lung cancer patients to assess their correlation with aetiological factors such as smoking or family history of cancer. We also analysed the contribution of genotypes CYP1A1 and GSTM1.

\section{Methods}

Formalin fixed paraffin wax embedded tissues of 35 pulmonary squamous cell carcinomas were obtained from the Saitama Medical School Hospital in Japan. Data on sex, age, occupational history, smoking history, and family history of cancer were obtained from patients' files. The mean (SD) age of the patients was 65 (7) years.

DNA was isolated from the $10 \mu \mathrm{m}$ deparaffinised sections according to a previously reported method. ${ }^{6}$ Exons $4-8$ of the p53 gene were amplified by nested polymerase chain reaction (PCR) method using two sets of primers for each exon. The PCR products underwent single strand conformation polymorphism (SSCP) analysis ${ }^{7}$ using silver staining for the detection of DNA mutations. PCR products which had abnormal shifted bands in SSCP were inserted into a plasmid vector using a TA cloning kit (Invitrogen, San Diego, CA), and sequencing analysis was performed using the Silver Sequence DNA sequencing system (Promega, Madison, WI).

Genotype CYP1A1 was analysed by PCRSSCP detection of the Ile $\rightarrow$ Val substitution. Briefly, genomic DNA was amplified using nested PCR with primers designed to include this mutation position; amplified fragments underwent SSCP analysis to detect CYP1A1 polymorphism. The bands detected in SSCP were identified by comparison with results of allele specific PCR on the same specimens.

Genotype GSTM1 was determined by differential PCR in which multiple genes are coamplified in the same reaction tube. PCP was performed using GSTM1 primers, p53 exon 6 primers, and CYP1A1 primers. Coamplification of p53 exon 6 and CYP1A1 gene that produce 216 and 106 base pair bands, respectively, were used as internal controls. Intact GSTM1 allele gave a 165 base pair 
Table 1 Patient data and results of analysis on $p 53$ gene mutations

\begin{tabular}{|c|c|c|c|c|c|c|}
\hline No & Sex & Age & Stage & Altered codon (exon) & Sequence change & Family history \\
\hline 1 & $\mathbf{M}$ & 58 & I & $291(8)$ & $\mathrm{AAG} \rightarrow \mathrm{AGG}$ & Sister (colon), father (stomach) \\
\hline 2 & $\mathrm{~F}$ & 43 & IV & $244(7)$ & $\mathrm{GGC} \rightarrow \mathrm{AGC}$ & \\
\hline 3 & $\mathrm{~F}$ & 62 & I & $192(6)$ & $\mathrm{CAG} \rightarrow \mathrm{CAA}$ & \\
\hline 4 & $\mathbf{M}$ & 76 & IIIA & $248(7)$ & $\mathrm{CGG} \rightarrow \mathrm{TGG}$ & \\
\hline 5 & M & 85 & $\mathrm{I}$ & $220(6)$ & $\mathrm{TAT} \rightarrow \mathrm{TGT}$ & \\
\hline 6 & $\mathbf{M}$ & 74 & I & $249(7)$ & $\mathrm{AGG} \rightarrow \mathrm{AGT}$ & Sister (stomach) \\
\hline 7 & $M$ & 76 & II & $195(6)$ & $\mathrm{ATC} \rightarrow \mathrm{TTC}$ & Father (liver) \\
\hline 8 & $\mathbf{M}$ & 68 & IIIB & $242(7)$ & $\mathrm{TGC} \rightarrow \mathrm{TTC}$ & Sister (colon) \\
\hline 9 & $\mathbf{M}$ & 68 & II & $242(7)$ & $\mathrm{TGC} \rightarrow \mathrm{TTC}$ & \\
\hline 10 & $\mathbf{M}$ & 70 & IIIA & $144(5)$ & $\mathrm{CAG} \rightarrow \mathrm{TAG}$ & Grandfather (oesophagus) \\
\hline 11 & M & 50 & IIIB & $234,244(7)$ & $\mathrm{TAC} \rightarrow \mathrm{TGC}, \mathrm{GGC} \rightarrow \mathrm{GGG}$ & \\
\hline 12 & $\mathbf{M}$ & 68 & II & $234(7)$ & $\mathrm{TAC} \rightarrow \mathrm{TGC}$ & Mother (liver) \\
\hline 13 & $\mathbf{M}$ & 46 & IIIA & $221-222(6)$ & del (AGCCG) & Sister (breast), father (liver ${ }^{\star}$, stomach) \\
\hline 14 & $\mathrm{~F}$ & 59 & IIIA & & & \\
\hline 15 & $\mathbf{M}$ & 61 & I & & & \\
\hline 16 & $\mathbf{M}$ & 64 & I & & & Sister (stomach) \\
\hline 17 & $\mathbf{M}$ & 62 & IIIA & & & \\
\hline 18 & $\mathbf{M}$ & 77 & II & & & \\
\hline 19 & $\mathbf{M}$ & 65 & I & & & \\
\hline 20 & M & 67 & IV & & & Sister (pancreas) \\
\hline 21 & $\mathbf{M}$ & 59 & IIIA & & & \\
\hline 22 & $M$ & 53 & II & & & \\
\hline 23 & M & 73 & I & & & \\
\hline 24 & $\mathrm{~F}$ & 67 & IIIB & & & Sister (uterus) \\
\hline 25 & $M$ & 58 & I & & & \\
\hline 26 & $\mathbf{M}$ & 76 & I & & & \\
\hline 27 & $M$ & 72 & I & & & \\
\hline 28 & $\mathbf{M}$ & 68 & II & & & \\
\hline 29 & $\mathbf{M}$ & 58 & I & & & \\
\hline 30 & $\mathbf{M}$ & 67 & I & & & \\
\hline 31 & $\mathbf{M}$ & 82 & IIIA & & & \\
\hline 32 & $\mathbf{M}$ & 62 & II & & & Father (oesophagus) \\
\hline 33 & $\mathbf{M}$ & 58 & II & & & Mother (uterus) \\
\hline 34 & $\mathbf{M}$ & 73 & II & & & \\
\hline 35 & $\mathbf{M}$ & 72 & I & & & \\
\hline
\end{tabular}

* A mutation of p53 gene in exon 5 was detected by SSCP analysis.

product and no visible bands were present in samples from homozygous deletion (GSTM1-/-). One allele deletion (+/-) was included in the " + " allele designation.

\section{Results}

In the present study, we focused on exons 4-8 of the p53 gene because $98 \%$ of base substitution mutations fall within these exons. Mutations were found in $13(37.1 \%)$ of 35 tumours through SSCP and these were confirmed by DNA sequencing. Of the 13 mutations, one was located in exon 5 , four in exon 6 , seven in exon 7, and one in exon 8 (table 1). One sample was found to carry two mutations, though only one of them produced an amino acid substitution (case 11). No abnormal bands were found using SSCP analysis for p53 gene of normal tissues. When p53 mutations were present, they were homogeneously distributed throughout the tumours.

Most of our patients had smoked except four patients and their mean (SD) smoking index was 49 (35) packs per year; however, the mutation positive group showed no relation with smoking index.

Hospital records revealed that seven $(53.8 \%)$ of the 13 patients with p53 mutations had a family history of cancer in first or second degree relatives, but only five $(22.7 \%)$ of the 22 patients without p53 mutations had a family history of cancer at the time of diagnosis (table 1). Although this difference was not statistically significant for all 13 patients, it became so when male patients were analysed separately ( $p<0.05$, odds ratio $7,95 \%$ confidence intervals (CI) 1.35-36). Anatomic sites of cancers in family members were variable (table 1).
These families were not considered to be associated with Li-Fraumeni syndrome. Furthermore, we analysed gastric carcinoma and $\triangle$ hepatoma tissue from the father of case 13 that $\overrightarrow{\overrightarrow{0}}$ was obtained in our hospital and p53 mutation in exon 5 was detected in hepatoma tissue.

For CYP1A1 genotypes provided by PCRSSCP, the frequencies of homozygote pre- $\frac{3}{0}$ dominant alleles (Ile/Ile), heterozygote alleles (Ile/Val), and homozygote rare alleles $(\mathrm{Val} / \mathrm{Val}) \stackrel{3}{3}$ were $48.6 \%$ ( 17 cases), $45.7 \%$ (16 cases), and $\delta$ $5.7 \%$ (two cases), respectively (table 2 ). The frequencies of GSTM1 null genotype was 윽 $54.3 \%$ (19 cases) (table 2). We found no $\frac{7}{0}$ significant relation between CYP1A1 or GSTM1 genotypes and p53 mutations, al- مै though GSTM1 genotypes in cases with family N history tended to be higher (nine of $12,75 \%$ ) N than cases without family history (10 of 23,0 $43.5 \%$ ). Also, no relation emerged between the genotypes and family history of cancer (table 2).

\section{Discussion}

Frequency of p53 gene mutation in this study $\frac{\mathbb{Q}}{\mathrm{Q}}$ was $37.1 \%$ (13 of 35 patients) in primary pulmonary squamous cell carcinomas, which is similar to other studies. ${ }^{12}$ All 13 cases in which shifted bands were detected by SSCP in exons 4-8 exhibited mutations by DNA sequence analysis. In our study, $61.5 \%$ (eight of 13 ) of p53 sequence abnormalities were G:C-A:T transitions, in line with Japanese patient mutational spectrum, ${ }^{1}$ while only $23 \%$ (three of 13 ) showed G:C-T:A transversions. G-T transversions have been reported to be significantly associated with lifetime cigarette consumption, ${ }^{12}$ and those 
Table 2 Frequencies of CYP1A1 and GSTM1 genotypes

\begin{tabular}{|c|c|c|c|c|c|c|}
\hline \multirow[b]{2}{*}{ Groups } & \multirow[b]{2}{*}{ No (\%) } & \multicolumn{3}{|l|}{$C Y P 1 A 1$} & \multicolumn{2}{|l|}{ GSTM1 } \\
\hline & & Ile/Ile & Ile/Val & Val/Val & + & - \\
\hline p53 mutation + & $13(100)$ & $7(53.8)$ & $6(46.2)$ & $0(0)$ & $8(61.5)$ & $5(38.5)$ \\
\hline p53 mutation - & $22(100)$ & $10(45.5)$ & $10(45.5)$ & $2(9.1)$ & $8(36.4)$ & $14(63.6)$ \\
\hline Family history + & $12(100)$ & $6(50.0)$ & $6(50.0)$ & $0(0)$ & $3(25.0)$ & $9(75.0)$ \\
\hline Family history - & $23(100)$ & $11(47.8)$ & $10(43.5)$ & $2(8.7)$ & $13(56.5)$ & $10(43.5)$ \\
\hline Total & $35(100)$ & $17(48.6)$ & $16(45.7)$ & $2(5.7)$ & $16(45.7)$ & $19(54.3)$ \\
\hline
\end{tabular}

,+ positive; -, negative. ciated with p53 mutations. We speculated that p53 mutations and family history of cancer may be linked to other factors. Ryberg et al reported that individuals homozygous for the GSTM1 null allele were at a higher risk of p53 mutation caused by PAHs. ${ }^{9}$ In addition, Kawajiri et al showed an association of CYP1A1 rare allele with mutations of p53 gene in lung cancer. ${ }^{10}$ Although the reason for discrepancies between our results and other studies is unknown, a possible explanation may be that most of our patients were heavy smokers so that carcinogen dose may have obscured effects of those genotypes on gene mutations.

In conclusion, our data suggest that p53 mutation in lung cancer patients might be associated with inherited characteristics of the patients leading to familial aggregation of cancer. However, genotypes of carcinogen metabolising enzymes CYP1A1 or GSTM1 were not related to $\mathrm{p} 53$ mutations.

Previous epidemiological studies have drawn attention to a tendency for familial aggregation among patients with lung cancer. ${ }^{8}$ The existence of familial susceptibility to cancer provides compelling evidence that genetic factors play an important role in the pathogenesis of lung cancer. In this study, male patients with p53 gene mutations showed a significantly higher frequency of family history of cancer compared with patients without $\mathrm{p} 53$ mutation (odds ratio $3.18,95 \%$ CI 1.35-36). In addition, the mutation spectrum did not reflect a carcinogen related G-T transversion. These findings suggest that p53 mutations in our cases might be associated with inherited characteristics rather than exogenous factors such as smoking.

Among factors that are known to be associated with lung cancer susceptibility, genotypes CYP1A1 and GSTM1 have been linked to a high risk of squamous cell carcinoma, especially in the Japanese population. ${ }^{35}$ We did not show any significant association between CYP1A1 or GSTM1 genotypes and p53 mutations or family history of cancer. On the contrary, the relation between GSTM1 genotypes and p53 mutations showed an inverse relation between those genotypes and family history. Although the number of cases was small, we concluded that CYP1A1 or GSTM1 genotypes were not asso-
1 Greenblatt MS, Bennett WP, Hollstein M, Harris CC. Mutations in the p53 tumor suppressor gene: Clue to cancer etiology and molecular pathogensis. Cancer Res 1994;54:4855-78.

2 Suzuki $H$, Takahashi T, Kuroishi T, Suyama M, Ariyoshi $Y$, Takahashi T, et al. P53 mutations in non-small cell lung cancer in Japan: Association betwe

3 Kawajiri K, Nakachi K, Imai K, Watanabe J, Hayashi S. The CYP1A1 gene and cancer susceptibility. Crit Rev Oncol Hematol 1993;14:77-87.

4 Drakoulis N, Cascorbi I, Brockmoller J, Gross CR, Roots I. Polymorphisms in the human CYP1Al gene as susceptibility factors for lung cancer: exon-7 mutation (4889A to $\mathrm{G}$ ), ity factors for lung cancer: exon-7 mutation (4889A to $\mathrm{G}$ ), and a $T$ to $C$ mu

5 Nakajima T, Elovava E, Auttila S, Hirvonen A, Camus AM, Hayes JD, et al. Expression and polymorphism of glutathione s-transferase in human lungs: risk factors in smoking-related lung cancer. Carcinogensis 1995;16:70711.

6 Ohshima S, Shimizu Y, Takahama M. Detection of C-Ki-ras gene mutation in paraffin sections of adenocarcinoma and gene mutation in parafin sections of adenocarcinoma and atypical bronchioloalveolar cell hyper

7 Murakami Y, Hayashi K, Sekiya T. Detection of aberrations of the p53 alleles and the gene transcript in human tumor cell lines by single strand conformation polymorphism analysis. Cancer Res 1991;51:3356-61.

8 Christine B, Ambrosone MS, Uma Rao, Michalek A, Cummings MK, Mettlinl CJ. Lung cancer histologic type and family history of cancer. Cancer 1993;72:1192-8.

9 Ryberg D, Kure E, Lystad S, Skaug V, Stangeland L, Mercy I, et al. P53 mutations in lung tumors: Relationship to , et al. P53 mutations in lung tumors: Relationship to
putative susceptibility markers for cancer. Cancer Res 1994; putative suscep

10 Kawajili K, Eguchi H, Nakachi K, Sekiya T, Yamamoto M. Association of CYP1A1 germline polymorphisms with mutation of the p53 gene in lung cancer. Cancer Res. 1996; $56: 72-6$ 